\title{
Microsampling for quantitative bioanalysis, an industry update: output from an AAPS/EBF survey
}

\author{
Shefali R Patel*,1, Peter Bryan², Neil Spooner ${ }^{3}$, Philip Timmerman ${ }^{4}$ \& Enaksha \\ Wickremsinhe ${ }^{5}$ \\ ${ }^{1}$ DMPK Department, Discovery Sciences, Janssen Research \& Development, LLC, 1400 McKean Road, Springhouse, PA 19477, USA \\ ${ }^{2}$ B2S Life Sciences, Mendham, NJ 07945, USA \\ ${ }^{3}$ Spooner Bioanalytical Solutions, Hertford, GG13 8BQ, UK \\ ${ }^{4}$ European Bioanalysis Forum, Brussels, Belgium \\ ${ }^{5}$ Lilly Research Laboratories, Eli Lilly \& Company, Indianapolis, IN 46285, USA \\ *Author for correspondence: Tel.: +1 215628 5371; spatel31@its.jnj.com
}

There is continuing interest in the development and application of various microsampling technologies for drug development. The AAPS bioanalytical community microsampling subgroup and the European Bioanalysis Forum conducted a survey of their members (39 individual organizations). This gives a snapshot of current practices and demonstrates that implementation of microsampling approaches is becoming increasingly commonplace, but not universal. Greater adoption was observed for nonclinical studies, particularly nonregulatory. A number of respondents reported that they have included microsampling data in regulatory submissions. Another important observation was that where microsampling is employed for clinical studies, dried blood approaches predominate, reflecting the interest in their use where they enable sample collection which is not feasible with standard approaches or to derive richer data sets.

First draft submitted: 30 January 2019; Accepted for publication: 28 February 2019; Published online: 11 April 2019

Keywords: AAPS • DBS • EBF • microsampling survey • quantitative bioanalysis

In the context of pharmaceutical drug development, microsampling is the collection of smaller biological matrix volumes (typically $\leq 50 \mu \mathrm{l}$ ) for the quantitative determination of circulating concentrations of drugs, metabolites and biomarkers than those by conventional approaches. After a decade of renewed focus, initially on dried blood spots (DBS) [1,2], microsampling continues to be of high interest for many bioanalytical scientists and end users of the concentration data reported by the bioanalytical laboratory. At the same time, given the perceived or real scientific, logistic and regulatory hurdles and complexity, the technology did not find its way into routine pharmaceutical $\mathrm{R} \& \mathrm{D}$ as was initially anticipated $[2,3]$. One of the potential reasons for this may be the confusion between DBS (one specific technology) and microsampling (which encompasses several technologies, typically sampling less than $50 \mu \mathrm{l}$ matrix, wet or dried) leading to the incorrect interpretation of the hurdles for one technology being projected onto all applications. It is therefore important to emphasize that microsampling is more than DBS.

Even though DBS sampling was the early eye catcher in this area, the implementation of microsampling has shifted to alternative sampling approaches, such as plasma microsampling and the development of a number of technologies for the simplified collection of fixed volume dried blood samples [4-9]. In turn, these approaches have enabled the areas for which microsampling is applied to become very broad. The focus in the nonclinical arena has emphasized the benefits in terms of the 3Rs (replace, reduce and refine experimental animal use) and/or improved pharmacokinetic $(\mathrm{PK})$ and pharmacodynamic $(\mathrm{PD})$ readouts [10,11]. Conversely, the applications of microsampling in clinical R\&D are very diverse. Sampling in standard Phase I-IV PK studies, enabling PK studies in children or neonates, simplifying sampling in remote areas and from critically ill patients, facilitating home sampling in postmarketing patient monitoring and enabling samples to be collected during clinical events, are only a few of the potential areas of application in pharmaceutical $R \& D$ today. Despite the large array of applications, the adoption 
of microsampling supporting clinical trials seems to have lagged, largely due to the conservative nature of drug development processes and their specific endpoints and regulations.

In 2012, the IQ Consortium Microsampling Group was formed to bring together the key experts in microsampling and share expertise, resulting in a publication outlining considerations when using microsampling for clinical drug development studies [12]. This group later transitioned to become the AAPS Microsampling Discussion Group (now known as the AAPS Bioanalytical Community Microsampling Subgroup). The European Bioanalysis Forum (EBF) established a liquid microsampling/dried blood spots (LMS-DBS) consortium in 2008 which has published regularly on their discussions [13-17]. Both groups (Microsampling Subgroup and the EBF) had efforts focused on sharing scientific and regulatory expertise and agreed that the time was right to get a snapshot of the current applications and experience of microsampling in the industry. Obtaining this information would be helpful in understanding which areas the technology has the best potential to add value. Hence, a survey of AAPS and EBF members was developed.

\section{Survey mechanics}

There have been a number of surveys on the use of microsampling for pharmaceutical drug development $[13,18-$ 20]. The first comprehensive North American survey on the pharmaceutical industry use of microsampling was sponsored by the IQ Consortium Microsampling Group in early 2012, and the survey was conducted within its members. The present survey was completed in December 2017 and was developed by the AAPS Microsampling Subgroup in collaboration with the EBF. This survey was sent to representatives from bioanalytical laboratories in the pharmaceutical industry and contract research organizations (CROs) worldwide to get a view on the current practices and hurdles related to microsampling technology, including questions on interactions with regulatory agencies.

In the current survey, questions were targeted separately at the use of DBS, liquid microsampling (using conventional approaches) and capillary liquid microsampling techniques, and how they are utilized in the different stages of drug development. The survey was divided into five categories: the current landscape, including responses for both dosed drug and biomarker analysis, regulations, including responses for both dosed drug and biomarker analysis, bridging studies, technology questions and questions on issues/hurdles encountered. The survey also enabled freeform comments to be submitted. The survey was distributed with instructions to limit responses to one response/organization/geography.

\section{Survey learnings}

The responses to the survey are set out in the Supplementary Material to this manuscript. A total of 39 independent organization responded to the survey (which represents majority of current industry users). The 39 survey respondents were evenly distributed between North America and Europe, with one response from Asia. The respondents were from a broad range of backgrounds, including large pharma (>10,000 employees; 46\%), medium to small pharma ( $<10,000$ employees, 18\%), large 'full service' CRO (18\%), small bioanalytical CRO (8\%) and biotech (5\%). All the respondents stated that they were engaged in conducting bioanalysis and greater than $85 \%$ stated they have performed bioanalysis across all development phases.

In interpreting the responses to the survey, we have defined a microsample as $\leq 50 \mu \mathrm{l}$ for approaches using liquid plasma and dried matrices and $\leq 64 \mu \mathrm{l}$ for those collecting liquid capillary blood, as this was how the respondents defined a microsample themselves (Supplementary Data, Q5-8 and Q17).

Where percentage values are given in this manuscript, this reflects a proportion of those that actively responded to the question and not of the total number of participants in the survey.

\section{Nonclinical}

It was notable that approximately half of the respondents were using microsampling to support nonclinical studies. Microsampling was used for both nonclinical blood and plasma collection either by liquid technique (capillaries or noncapillaries) or by dry technique (DBS or volumetric absorptive microsampling [VAMS] - the latter being a commercialized approach for the collection of a fixed volume of dried matrix).

Of the survey participants, $76 \%$ responded to the questions on the use of microsampling for the determination of nonclinical PK. The data demonstrated that plasma microsampling is more prevalent than that of blood among the respondents for nonclinical studies. 
Further, the survey showed that:

- Plasma - the use of liquid capillaries was more prevalent (47\%) than other liquid (40\%) or dried techniques $(14 \%)$;

- Blood - capillary and dried approaches were employed by a similar number of respondents (34\%) and were more frequently used than other liquid techniques (18\%);

- Dried blood - the use of DBS and VAMS were similar.

For the nonclinical studies supported by microsampling, regardless of technique or matrix, discovery studies accounted for the higher percentage (18-47\%), non-GLP studies were intermediate (10-38\%) and GLP studies accounted for the lower percentage $(0-28 \%)$. Only one respondent used a microsampling approach for the determination of PK in matrices other than blood or plasma.

Of the survey participants, $69 \%$ responded to the questions on the use of microsampling for the determination of nonclinical biomarkers. Compared with PK sampling, fewer organizations have supported nonclinical biomarker analysis with microsampling. No respondents used a microsampling approach for the determination of biomarkers in matrices other than blood or plasma.

For nonclinical studies, both blood and plasma microsampling approaches have been used to generate data used in files submitted to regulatory authorities, including the US FDA, European Medicines Agency, and Medicines and Healthcare Products Regulatory Agency. These questions were responded by $59 \%$ of the survey participants for PK. Approximately two-thirds of respondents have included microsampling PK data from discovery, non-GLP rodent or non-GLP in other species studies in their regulatory submissions. Conversely, less than half have submitted data from GLP studies in rodents or other species. Further, the majority of these submitted studies used plasma PK data (36-53\%) compared with blood data (17-38\%), while up to $17 \%$ submitted both plasma and blood PK data to regulators.

It is notable that greater than half of respondents have included rodent microsampling PK data from GLP studies in their IND submissions and that a quarter of respondents have included rodent and nonrodent microsampling data from GLP studies in their NDA submissions.

For nonclinical biomarker studies, the number of respondents was lower (15 respondents). Of these, a few had included biomarker microsampling data in a regulatory submission derived from blood (three respondents), or plasma (three respondents). However, at least two of these respondents had included microsampling biomarker data derived from GLP studies in their submissions. One respondent had included nonclinical biomarker microsampling data in an NDA submission.

For microsampling studies which have been submitted to health authorities, the agencies have been requesting bridging studies between conventional and microsampling. For studies which had supported the blood PK data, conventional plasma PK as well as satellite/bridging studies were done to support the IND or NDA, as well as for internal scientific purposes.

The smallest volumes used during nonclinical studies, as reported by the respondents were $\leq 25 \mu$ l for wet plasma and $\leq 32 \mu \mathrm{l}$ for wet blood. For DBS, the preference for $20 \mu \mathrm{l}$ was slightly higher than using $10 \mu \mathrm{l}$ volume, while for VAMS it was the other way around.

\section{Clinical}

Of the survey participants, $89 \%$ responded to the questions on the use of microsampling for the determination of clinical PK. The adoption of microsampling to support PK sampling during the conduct of clinical trials (across Phase I first in human to late clinical Phase IV studies) amongst respondents was not high (30\%). The survey suggests that the use of dried blood (DBS and VAMS) and liquid plasma microsampling techniques were more or less evenly distributed and were utilized more than liquid blood, or dried plasma. No respondents had utilized microsampling for the collection of other matrices, such as urine.

Of the survey participants, approximately half responded to the questions on the use of microsampling for the determination of clinical biomarkers. A similar trend was evident for collection of biomarker samples across clinical trials to that observed for clinical PK microsampling. However, the use of microsampling for biomarkers was low (less than 20\%).

For clinical studies, only a small proportion of respondents have included microsampling PK data in their submissions to regulators. 
The breakdown was as follows:

- First in human - blood $18 \%$, plasma $9 \%$;

- Phase II - blood 33\%, plasma 0\%;

- Late phase volunteer - blood $0 \%$, plasma $0 \%$;

- Late phase patient/special populations - blood 25\%, plasma 9\%.

These respondents represented 59\% of those taking part in the survey. Only one respondent stated that they have included microsampling PK data from late phase/special populations studies in an NDA submission.

For clinical studies where microsampling was used, $90 \%$ of the respondents stated that the samples were collected by a healthcare professional, rather than self-sampled (67\% of participants responded).

\section{Technical considerations}

For the blood capillary microsampling technique, out of the 16 respondents using this approach, the majority (44\%) analyzed the contents of the entire capillary, while a similar number (38\%) transferred the sample to a small microvessel and the remainder sampled the original capillary with another accurate volume capillary. A further 21 respondents stated that they did not use this approach for microsampling.

For plasma capillary microsampling, half of respondents using this approach brought the plasma into a smaller microvessel, while smaller numbers sampled the original capillary with another accurate volume capillary (29\%) or analyzed the whole capillary $(21 \%)$. It is notable that 14 respondents stated that they did not use this approach for microsampling.

Approximately two-thirds of the survey respondents stated that they are diluting their plasma and blood capillary microsamples. When diluting plasma microsamples there was an even split between the use of plasma or buffer, compared with $86 \%$ using plasma to dilute conventional plasma samples. For capillary blood microsampling, buffer was used by half of the respondents, while there was an even split of the remainder between plasma and phosphate buffered saline as diluents.

For dried blood microsampling approaches, of the 26 respondents, half declared that they have standardized on the VAMS sampler. Smaller numbers used DBS techniques, with an equal split (approximately 25\% each) between spotting an approximate volume and taking an accurate subsample for analysis and spotting an accurate volume onto the substrate and analyzing the entire spot. A few respondents indicated they sampled an accurate volume of blood with a pipette from a microtube or sampled accurately with a fixed capillary.

The survey participants were asked to share their major challenges for each of the microsampling approaches. For DBS sampling, the highest response was around hematocrit issues, which may explain the preference in using the VAMS approach to obtain a fixed volume sample. The other issues identified by respondents were the quality of samples (homogeneity, accurate volumes, double spotting and clotted samples), recovery with spot aging, additional work for the bioanalytical laboratory and regulatory acceptance of the approach. For capillary liquid microsampling, the major issue was around blood volume (hemolysis, plasma yield, metabolite identification, repeats, incurred sample reanalysis and appropriate labware). A number of respondents also stated that assay sensitivity and recovery of the analyte from the capillary were important issues. The issues encountered for liquid microsampling using conventional technologies were similar to those for capillary liquid microsampling with blood volume issues being the most frequent. Other issues were assay sensitivity and the accuracy of sample aliquoting. A major issue for all the techniques was concerns over the acceptance of data derived from these approaches by the health authorities and translation of the data to clinical studies.

\section{Discussion}

Nonclinical

Results of the survey indicate that some general trends in the use of microsampling techniques for nonclinical studies are apparent. Approaching 50\% of respondents have used microsampling for nonclinical studies, which is encouraging, although not as high as might be hoped when considering the ethical benefits (the 3R's) of implementing this approach and the length of time the bioanalytical community has been discussing this approach and for which viable solutions have been readily available.

It is notable from the survey responses that plasma is favored over blood as a matrix, while liquid samples are preferred over dried. The potential reasons for this are that plasma is a more familiar matrix for most organizations. 


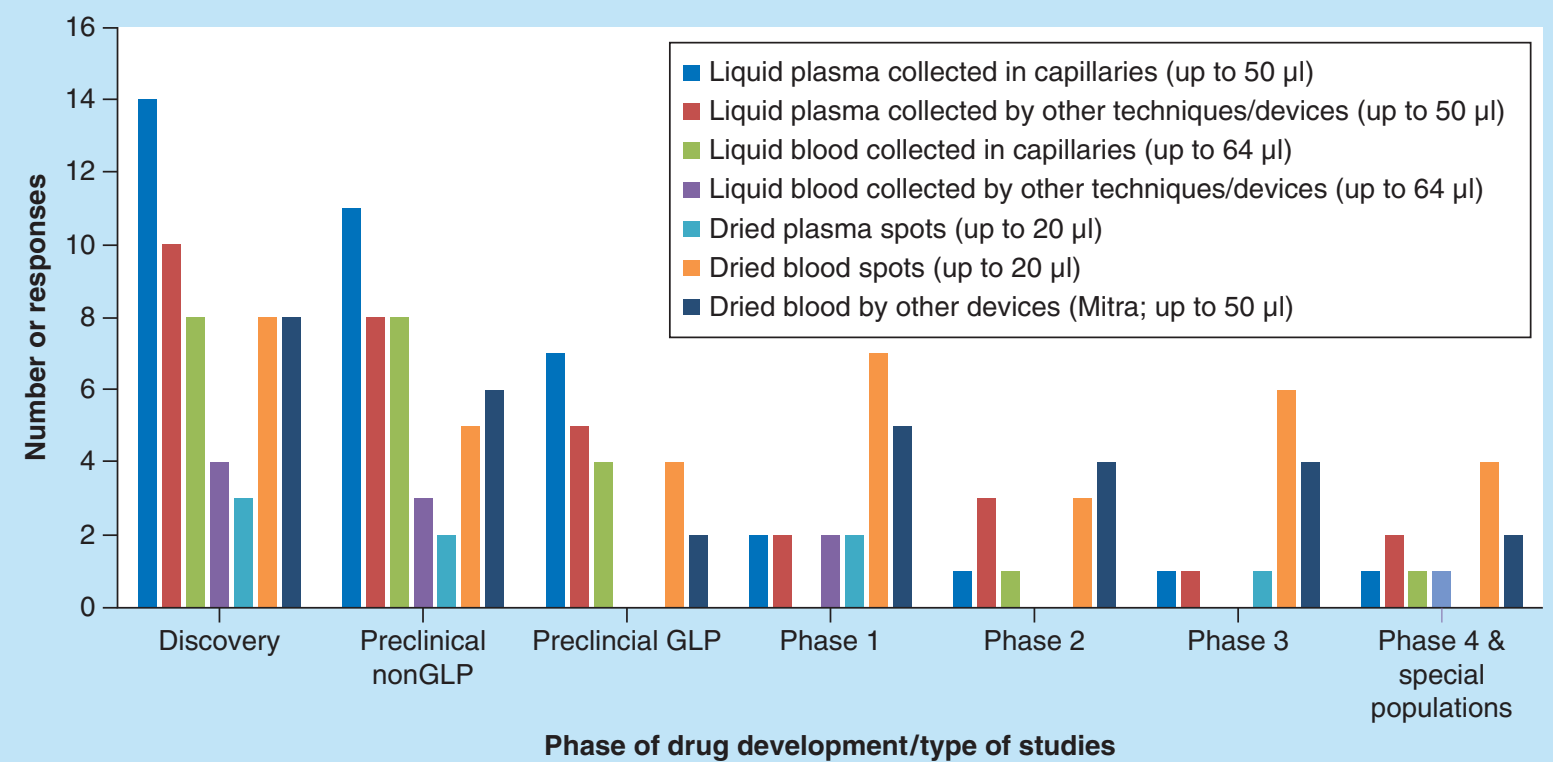

Figure 1. Utilization of different microsampling approaches for studies at various stages of drug development. The data represents responses from 39 different pharmaceutical companies and contract research organizations.

In addition, liquid matrices are more likely to fit into existing analytical workflows and the remaining samples can be more readily used for other purposes, such as reassay, the identification of metabolites and the study of biomarkers. Further, as many early phase nonclinical studies are small studies and are often performed in close proximity to the site of bioanalysis, the benefits of dried samples (reduced shipping costs, increased stability for some analytes) are not so readily realized. In addition, dried sample methods tend to require more method development and have nonstandard bioanalytical workflows compared with liquid matrices. These reasons may also explain why the use of microsampling was more prevalent for the determination of PK than biomarkers for these studies and why microsampling is not a more widely adopted approach.

An important observation was that microsampling approaches are more frequently being employed in discovery phase projects than later phase non-GLP and GLP studies (Figure 1). The potential reasons for fewer later phase studies using this approach is likely to be due to a number of factors, including: the slow progression of drug candidates through development, high attrition rates, a reluctance for organizations to change from well-established and proven blood sampling approaches, and a concern that data will not be accepted by regulatory bodies. The latter observation is supported by the fact that many organizations have not yet submitted regulatory filings which contain nonclinical microsampling PK data and even fewer have submitted microsampling biomarker data.

As the benefits of microsampling technologies become more widely recognized and the associated technologies become more broadly accepted and routine to use, it is likely that this approach will become more acceptable by organizations for use in nonclinical GLP studies. In turn, this may lead to more studies using these techniques being filed with regulatory authorities. However, it appears that progress to widespread implementation is likely to be slow.

\section{Clinical}

From the survey results, it has been difficult to tease out the intricacies intended to be disseminated from the survey. Adoption of microsampling has been slow probably due to the perceived uncertainty of the acceptance of the technique by regulatory agencies. Far fewer studies have been performed in the clinical phase than in the nonclinical phase, and the majority of respondents were not using microsampling for clinical studies (Figure 1). However, based on recent conferences, presentations and publications, there has clearly been a continuing interest in the adoption of microsampling for clinical studies. As many as 33\% of the respondents have submitted early phase clinical data to regulatory agencies, and as many as $25 \%$ have submitted late phase clinical data from patients 
and special populations. It appears that the pharmaceutical industry is finding the appropriate use of the technique, and the proposed advantages of microsampling have been proven. For example, VAMS has shown the ability to mitigate the hematocrit effects associated with DBS sampling [6,21]. As many as 30\% of respondents had used either DBS or VAMS across all phases of clinical development, with VAMS being slightly more commonly used than DBS and both dried blood techniques being more commonly employed compared with liquid microsampling approaches (Figure 1). The latter finding is indicative that organizations are starting to realize not only the benefits of smaller volumes, but also the convenience and potential cost savings of dried blood approaches.

Liquid plasma microsamples were collected more often than liquid blood microsamples, probably reflecting an organizations familiarity with this matrix in clinical studies. The DBS samples were collected more often than dried plasma spots, which is probably due to the DBS approach offering fewer sample processing steps, for example, the preparation of plasma is not required prior to spotting. According to respondents, other matrices (e.g., urine) were not collected by microsampling techniques. A few respondents used microsampling for biomarker sample collection, but the techniques were not commonly used.

The key takeaway from the survey was that microsampling is increasingly being integrated into clinical development with higher adoption during early phase clinical studies and in studies with special patient populations. Bridging studies were performed to support the introduction of a new/different matrix. Samples were predominantly being collected by healthcare professionals (therefore most likely at the clinical sites) and not by the patient (home sampling). The survey also showed that regulatory submissions supported by microsampling have been submitted to major regulatory agencies (FDA, European Medicines Agency and Healthcare Products Regulatory Agency).

\section{Technical considerations}

For plasma and blood microsampling, it is apparent that there is no consensus approach as to how the downstream processing of these samples should be performed, including subsampling and dilution. This is probably reflective of the fact that it is still relatively early days in the adoption of this approach for sample collection and quantitative bioanalysis. Further, it is also potentially due to the small number of laboratories currently using this approach only 28 out of the 39 survey participants were using capillary plasma microsampling and 16 were using blood.

For dried blood microsampling approaches, it is notable that half are using the VAMS sampler, which is a relatively recent introduction. Where DBS sampling is used, there was no consensus as to how the sample was spotted. Again, as with capillary microsampling, this is probably reflective of the relatively recent introduction of these technologies into sample collection and bioanalytical workflows and the limited number of practitioners.

The survey respondents listed a number of issues with the various microsampling approaches (Supplementary Material). Many of these were related to concerns over the quality of the sample obtained and hence the data generated. It is hoped that increased investigation of these issues and information sharing by users will lead to more adoption of best practice and standardized approaches, which will alleviate these concerns. Another major apprehension was around assay sensitivity associated with the small sample volumes. Historically, the sensitivity of the detectors used for bioanalytical analyses have increased and the workflows improved, the continued progression of which will make this a less important issue in the future.

\section{Future perspective}

Nonclinical

Much needs to be done to make microsampling technologies more widely acceptable by both industry and regulators, and the diversity in such techniques will continue to evolve in the nonclinical space. Pharmaceutical companies, CROs and vendors need to work out and publicize streamlined and standardized microsampling processes that are geared for nonclinical sampling. The recent publication of the Q\&As for microsampling as an addendum to the ICH S3A, provides guidance including the expectation by the regulatory agencies to establish concordance (bridging) between microsampling and conventional sampling and hence, this will continue to be an expectation of the regulatory agencies [22]. There is a concern that this requirement is working against the widespread adoption of microsampling for nonclinical studies, as it results in additional analytical methods to be developed, the number of species to be tested and does not deliver the ethical (3Rs) benefits that microsampling is intended to provide. It is hoped that further standardization and better understanding of microsampling techniques will provide a framework to accept microsampling, as is the case for current regular plasma sampling. 


\section{Clinical}

Significant improvements continue to be made in developing microsampling techniques geared toward enabling and supporting the collection of smaller volumes of blood. The recent US FDA guidance on Bioanalytical Method Validation Guidance for Industry (May 2018) discusses 'dried blood spots' (which extends to all microsampling techniques) in the context of its benefits ('reduced blood sample volumes collected for drug analysis as well as ease of collection, storage and transportation') but also requires the conduct of 'correlative studies with traditional sampling during drug development' [23]. It is hoped that microsampling data supporting regulatory submissions will be accepted by governing agencies, without the need for exhaustive bridging studies or without the need for bridging to traditional plasma data, encouraging and facilitating the adoption of such techniques globally. A reassuring aspect of the new guidance is that 'sponsors are encouraged to seek feedback from the appropriate US FDA review division early in drug development', where discussion can include the need for bridging concentration data between microsampling and traditional sampling.

There may even be the day when sparse sampling, using microsampling, could be performed on all subjects in late phase studies. Certainly, microsample collection of more biomarkers and biotherapeutics (in addition to small molecule drugs), and immunogenicity sampling with minimally invasive techniques is a lofty goal which would have positive effects on the healthcare industry and patient health in the future.

\section{Home sampling}

The collection of blood samples at home (not readily possible with current standard blood sampling procedures) is attractive for a number of reasons, in addition to those set out for routine clinical trial use. These include the ability to collect richer PK and biomarker datasets, with a larger number of data points. Further, they offer the possibility of helping to demonstrate patient compliance in taking their medication, provide a means of setting the correct dose and enabling samples to be collected in close temporal proximity to a clinical event, for example migraine [24]. The over-riding benefit of this approach is that the patient (and potentially their caregiver) is not inconvenienced by having to travel to a blood collection clinic that is often remote from their home.

Whether these samples are collected by the patient themselves, by a caregiver or by a healthcare professional, it is apparent that the technologies employed need to be robust, simple, logical to use, nonobtrusive and provide a means of obtaining blood that is acceptable to the patient in terms of pain and complexity. Further, high quality training in the means of sample collection needs to be provided in a timely and ongoing manner with appropriate safeguards built into the technologies, including workflows, to ensure sample quality is paramount [24-26]. This will enable a sample of the required quality to be collected and preserved for analysis.

Another important consideration for the development of future patient centric blood sampling technologies is their ready integration into analytical workflows. Many laboratories will be eager for such technologies to fit into existing established processes, while others will embrace new analytical workflows, particularly if increased automation and enhanced data quality is provided by their adoption.

\section{Conclusion}

The results of the survey performed by the AAPS Bioanalytical Community Microsampling Subgroup and the EBF give us an important snapshot of the current status for the implementation of microsampling approaches by pharmaceutical companies and CROs. It is apparent from the survey results that while the implementation of these technologies is not comprehensive, it is clearly reasonably widespread and ongoing. This probably reflects the reasoning of most organizations, that microsampling is a developing and important approach that is best reserved for and should be more readily applied in applications where it adds value compared with standard blood collection and analysis approaches, for example 3Rs, home sampling, remote sample collection, pediatrics, critically ill, obtaining richer data sets, obtaining data during clinical episodes - among others. However, there is a concern that the requirement of regulators that sponsors provide comparison data between microsampling approaches and more conventional sampling, for both nonclinical and clinical studies, is stifling innovation and implementation of these technologies. In turn, this nonscientific requirement for comparison data is preventing the realization of the obvious ethical and other benefits that these technologies offer. As an industry, we should continue to focus our efforts on enabling the development of microsampling approaches in those areas where they bring benefit. Further, we should actively collaborate across organizations, including beyond pharmaceutical drug development and publish the outcomes of our studies to continue with the path forward. 


\section{Executive summary}

Background

- Microsampling continues to be of high interest for many bioanalytical scientists and end users of the concentration data reported by the bioanalytical laboratory.

- A survey was developed by the AAPS Bioanalytical Community Microsampling subgroup in collaboration with the European Bioanalysis Forum, to get a view on the current practices and hurdles related to microsampling technology, including questions on interactions with regulatory agencies. This was completed by 39 representatives from bioanalytical laboratories in the pharmaceutical industry and contract research organizations worldwide.

- It is important to emphasize that while dried blood spot sampling is a common microsampling approach, the term microsampling is also applied to a wide variety of technologies.

Discussion

- The results indicate that while the utilization of microsampling sample collection for quantitative bioanalysis is common, it is far from being universal.

- Microsampling is more routinely adopted for nonclinical studies than clinical.

- For nonclinical studies, microsampling approaches are more frequently being employed in discovery phase projects than later phase non-GLP and GLP studies.

- For clinical studies, microsampling is increasingly being integrated into clinical development with higher adoption during early phase clinical studies and in studies with special patient populations (pediatrics, critically ill), or where samples are collected away from the clinic (remote areas, home sampling) or during clinical episodes, and other scenarios, rather than for standard pharmacokinetic analyses.

- Liquid blood microsampling techniques are favored for nonclinical studies, while dried blood approaches are favored for clinical studies.

- Overall, adoption of microsampling has been slow, probably due to the perceived uncertainty of the acceptance of the technique by regulatory agencies.

- A notable number of respondents have submitted nonclinical and clinical microsampling data to a variety of regulatory agencies.

Future perspective

- In both the nonclinical and clinical arena, much needs to be done to make microsampling technologies more widely acceptable by both industry and regulators, and the diversity in such techniques will continue to evolve in the nonclinical space.

- Significant improvements continue to be made in developing microsampling techniques geared toward enabling and supporting the collection of high quality smaller volumes of blood.

- Another important consideration for the development of future patient centric blood sampling technologies is their ready integration into analytical workflows and increasing need for automation.

\section{Conclusion}

- It is apparent from the survey results that while the implementation of these technologies is not comprehensive, it is clearly reasonably widespread and ongoing.

- As an industry, we should continue to focus our efforts on enabling the development of microsampling approaches in those areas where they bring benefit.

Supplementary data

See online at: www.future-science.com/doi/10.4155/bio-2019-0019

\section{Acknowledgements}

The authors would like to thank all the members of the AAPS Microsampling Discussion Group, IQ Drug Metabolism Leadership Group and the EBF that completed the survey.

Financial \& competing interests disclosure

The authors have no relevant affiliations or financial involvement with any organization or entity with a financial interest in or financial conflict with the subject matter or materials discussed in the manuscript. This includes employment, consultancies, honoraria, stock ownership or options, expert testimony, grants or patents received or pending, or royalties.

No writing assistance was utilized in the production of this manuscript. 


\section{References}

Papers of special note have been highlighted as: $\bullet \bullet$ of considerable interest

1. Beaudette P, Bateman K. Discovery stage pharmacokinetics using dried blood spots. J. Chromatogr. B Analyt. Technol. Biomed. Life Sci. 809(1), 153-158 (2004).

2. Spooner N, Barfield M. Dried blood spots as a sample collection technique for the determination of pharmacokinetics in clinical studies: considerations for the validation of a quantitative bioanalytical method. Anal. Chem. 81(4), 1557-1563 (2009).

3. Spooner N. Dried blood spot sampling for quantitative bioanalysis: time for a revolution? Bioanalysis 2(11), 1781 (2010).

4. Jonsson O, Villar R, Nilsson L et al. Capillary microsampling of $25 \mu \mathrm{l}$ blood for the determination of toxicokinetic parameters in regulatory studies in animals. Bioanalysis 4(6), 661-674 (2012).

5. Bowen C, Licea-Perez H, Karlinsey MZ et al. A novel approach to capillary plasma microsampling for quantitative bioanalysis. Bioanalysis 5(9), 1131-1135 (2013).

6. Denniff P, Spooner N. Volumetric absorptive microsampling: a dried sample collection technique for quantitative bioanalysis. Anal. Chem. 86(16), 8489-8495 (2014).

7. Lenk G, Sandkvist S, Pohanka A et al. A disposable sampling device to collect volume-measured DBS directly from a fingerprick onto DBS paper. Bioanalysis 7(16), 2085-2094 (2015).

8. Mengerink Y, Mommers J, Qiu J et al. A new DBS card with spot sizes independent of the hematocrit value of blood. Bioanalysis 7(16), 2095-2104 (2015).

9. Leuthold L, Heudi O, Deglon J et al. New microfluidic-based sampling procedure for overcoming the hematocrit problem associated with dried blood spot analysis. Anal. Chem. 87(4), 2068-2071 (2015).

10. Chapman K, Burnett J, Corvaro M et al. Reducing preclinical blood volumes for toxicokinetics: toxicologists, pathologists and bioanalysts unite. Bioanalysis 6(22), 2965-2968 (2014).

11. Wickremsinhe E, Perkins E. Using dried blood spot sampling to improve data quality and reduce animal use in mouse pharmacokinetic studies. J. Am. Assoc. Lab. Anim. Sci. 54(2), 139-144 (2015).

12. Evans $\mathrm{C}$, Arnold M, Bryan P et al. Implementing dried blood spot sampling for clinical pharmacokinetic determinations: considerations from the IQ Consortium Microsampling Working Group. AAPS J. 17(2), 292-300 (2015).

-. Comprehensive overview on implementation of microsampling for clinical drug development studies.

13. Abbott R, Smeraglia J, White $S$ et al. Conference Report - European Bioanalysis Forum Workshop: implementing dried blood spot sampling for clinical pharmacokinetic determinations. Bioanalysis 2(11), 1809-1816 (2010).

14. Timmerman P, White S, Globig S et al. EBF recommendation on the validation of bioanalytical methods for dried blood spots. Bioanalysis 3(14), 1567-1575 (2011).

15. Timmerman $\mathrm{P}$, White $\mathrm{S}$, Cobb Z et al. Update of the EBF recommendation for the use of DBS in regulated bioanalysis integrating the conclusions from the EBF DBS-microsampling consortium. Bioanalysis 5(17), 2129-2136 (2013).

16. Cobb Z, Roohde M, Love I et al. Feedback from the EBF liquid micro-sampling consortium: capillary liquid micro-sampling and the homogeneity of the resultant samples. Bioanalysis11(6), 525-532 (2019).

17. Hawthorne G, Dillen L, Barfield M et al. Feedback from the EBF liquid micro sampling consortium: microsampling: assessing accuracy and precision of hand held pipettes and capillaries. Bioanalysis 11(6), 533-542 (2019).

18. Chapman K, Chivers S, Gliddon D et al. Overcoming the barriers to the uptake of nonclinical microsampling in regulatory safety studies. Drug Discov. Today 19(5), 528-532 (2014).

19. Denniff P, Holliman C, Svensson L, Weng N, Patel S. Bioanalysis Zone: DBS survey results. Bioanalysis 6(3), 287-291 (2014).

20. Bioanalysis Zone. Microsampling 2 years on. www.bioanalysis-zone.com/2016/10/17/microsampling-2-years/

-. Regulatory document outlining approaches and considerations for the use of microsampling for the determination of toxicokinetics (TK) in drug development toxicology studies.

21. Spooner N, Denniff P, Michielsen L et al. A device for dried blood microsampling in quantitative bioanalysis: overcoming the issues associated blood hematocrit. Bioanalysis 7(6), 653-659 (2015).

22. Focus on Microsampling. Questions and answers to ICH S3A: note for guidance on toxicokinetics: the assessment of systemic exposure in toxicity studies. ICH Harmonised Guidelines (2017). www.ich.org/fileadmin/Public_Web_Site/ICH_Products/Guidelines/Safety/S3A/S3AIWG_Step4_2017_1116.pdf

23. Bioanalytical Method Validation: Guidance for Industry. US Department of Health and Human Services, Food and Drug Administration, Center for Drug Evaluation and Research, Center for Veterinary Medicine (2018). www.fda.gov/downloads/Drugs/Guidance/ucm070107

24. Li CC, Dockendorf M, Kowalski K et al. Population PK analyses of ubrogepant (MK-1602), a CGRP receptor antagonist: enriching in-clinic plasma pk sampling with outpatient dried blood spot sampling. J. Clin. Pharmacol. doi:10.1002/jcph.1021 (2017). (Epub ahead of print). 
Special Report Patel, Bryan, Spooner, Timmerman \& Wickremsinhe

25. Panchal T, Spooner N, Barfield M. Ensuring the collection of high-quality dried blood spot samples across multisite clinical studies. Bioanalysis 9(2), 209-213 (2017).

26. Lelong C, Wickremsinhe E, Filali-Ansary, Spooner N. Training and microsample collection. In: Microsampling in Pharmaceutical Bioanalysis Future Science, London, UK, 1, 15-28 (2013). 